\title{
Admission policies and methods at crossroads: a review of medical school admission policies and methods in seven Asian countries
}

\author{
Diantha Soemantri ${ }^{1}$ Indika Karunathilake ${ }^{2}$, Jen-Hung Yang, ${ }^{3,4}$, Shan-Chwen Chang ${ }^{5}$, Chyi-Her Lin ${ }^{6}$, \\ Vishna D. Nadarajah ${ }^{7}$, Hiroshi Nishigori ${ }^{8}$, Dujeepa D. Samarasekera ${ }^{9}$, Shuh Shing Lee ${ }^{9}$, Lilybeth R. Tanchoco ${ }^{10,11}$ \\ and Gominda Ponnamperuma ${ }^{2}$ \\ ${ }^{1}$ Department of Medical Education, Faculty of Medicine Universitas Indonesia, Jakarta, Indonesia, ${ }^{2}$ Department \\ of Medical Education, University of Colombo, Colombo, Sri Lanka, ${ }^{3}$ Department of Medical Education, Changhua \\ Christian Hospital, Changhua, ${ }^{4}$ School of Medicine of Kaohsiung Medical University, Kaohsiung, ${ }^{5}$ National Taiwan \\ University College of Medicine, Taipei, ${ }^{6}$ College of Medicine, I-Shou University, Kaohsiung, Taiwan, ${ }^{7}$ International \\ Medical University, Kuala Lumpur, Malaysia, ${ }^{8}$ Center for Medical Education, Nagoya University, Nagoya, Japan, \\ ${ }^{9}$ Center for Medical Education, National University of Singapore, Singapore, and Departments of ${ }^{10}$ Physiology \\ and ${ }^{11}$ Anesthesiology, Manila Central University College of Medicine, Caloocan City, Philippines
}

Selecting the right applicants is an important part of medical student admission. While one universally accepted selection criterion is academic capacity, there are other criteria such as communication skills and local criteria (e.g., socio-cultural values) that are no less important. This article reviews the policies and methods of selection to medical schools in seven countries with varying socio-economic conditions and healthcare systems. Senior academics involved in medical education in Indonesia, Japan, Malaysia, the Philippines, Singapore, Sri Lanka, and Taiwan completed a pre-agreed pro-forma per each country to describe the country's admission policies and methods. The details were then compared and contrasted. This review identifies tension between many of the policies and methods used in medical school admissions, such as between the need to assess non-cognitive abilities and widen access, and between the need for more medical professionals and the requirement to set high entry standards. Finding the right balance requires careful consideration of all variables, including the country's human resource needs; socio-economic status; graduates' expected competencies; and the school's vision, mission, and availability of resources.

Key Words: Student selection, Medical students, Asians

\section{Introduction}

Admission to medical school in almost all countries around the world is highly sought after; hence, it is highly competitive [1]. Amidst such competition, the admission or selection processes, as the gatekeeper of the profession, have an important obligation to admit the right applicants in order to maintain the society's healthcare standards.

The 2018 Ottawa Consensus Statement on selection highlighted the need for medical education research to
Received: March 2, 2020 • Revised: May 24, 2020 • Accepted: June 3, 2020 Corresponding Author: Diantha Soemantri (https://orcid.org/0000-0002-3956-1265) Department of Medical Education, Faculty of Medicine Universitas Indonesia, Salemba Raya 6, Jakarta Pusat 10430, Indonesia

Tel: +62.21.3901814 email: diantha.soemantri@ui.ac.id, diantha.soemantri@gmail.com

*This study was presented at the 12th Jakarta Meeting on Medical Education organized by Faculty of Medicine Universitas Indonesia, 19-20 October 2019.
Korean J Med Educ 2020 Sep; 32(3): 243-256.

https://doi.org/10.3946/kjme.2020.169

eISSN: 2005-7288

(C) The Korean Society of Medical Education. All rights reserved. This is an open-access article distributed under the terms of the Creative Commons Attribution Non-Commercial License (http:// creativecommons.org/licenses/by-nc/3.0/), which permits unrestricted non-commercial use, distribution, and reproduction in any medium, provided the original work is properly cited. 
go beyond focusing on studying methods or tools of selection towards selection policies [2]. Roberts and Prideaux [3] also argued that research on selection should be geared towards understanding the impact of the socio-cultural elements in the selection process. This is particularly important because the selection is inevitably driven by local policies relating to widening access, workforce issues, and the financial capabilities of medical schools. While this makes research in the local and regional context important, to date, the literature on how best to align policies and methods of selection is limited.

Admission policy is a set of principles or approaches that the authorities (e.g., governments) expect all stakeholders to follow. Policies are adopted based on universal and local criteria [3]. The universal criteria are applicable anywhere in the world and the most common are academic or intellectual capacity and the potential to master professional and other non-technical skills. Local criteria, however, are dependent on the needs, capacity (e.g., available resources), and socio-cultural values of a country. For example, if a country considers it important to have medical professionals representing all sociodemographic strata of the society, then such a country may adopt a selection system to ensure all strata, including socioeconomically underprivileged segments in society, have a fair opportunity to enter the medical school (e.g., [4,5]). Hence, the country could implement various affirmative action and positive discrimination programmes, including quota systems, to widen access and ensure the entry of certain groups to medical schools.

Admission methods rely on individual assessments (e.g., secondary school leaving examinations, admission interviews, aptitude tests) to select medical students. Ideally, the methods should follow the policies. If the example above is extended to identify admission methods, the example country needs to adopt admission methods that would be fair to all segments of the society. However, the identification of these methods may also be dependent on various other factors, such as the availability of resources and the know-how.

As such, the policies and methods used to select medical students vary from country to country. These differences are usually due to underlying contextual issues, which have been poorly explored and hence poorly understood, particularly in the international setting. The aim of this study was to review the admission policies and methods of medical schools in seven countries with widely varying socio-economic conditions and healthcare systems. In doing so, this review aims to identify common issues related to admission policies and methods that the countries encounter and discuss the mechanisms adopted to resolve these issues. Finally, based on the experiences of these countries, some broad guidelines to mitigating some of the common issues related to admission policies and methods are delineated.

\section{Methods}

We identified seven Asian countries (Indonesia, Japan, Malaysia, the Philippines, Singapore, Sri Lanka, and Taiwan) to collect data on the admission policies and methods adopted in each country. These seven countries were primarily selected based on the feasibility of access to their admission policies and methods. However, the said countries respresented a wide array of not only educational and healthcare systems, but also socioeconomic, cultural, and demographic backgrounds in the Asia Pacific region. This way, the reviewers ensured maximum variability of data that is essential for a qualitative investigation. The diverse backgrounds of 
these countries are shown by the following indicators obtained from The World Bank. The total population of each country in 2018 varied, from the lowest which is Singapore (5.7 million) to Indonesia as the highest (267 million). The gross domestic product ranges from 88.9 billion US dollar (Sri Lanka) to 4.971 trillion (Japan) in 2018, whereas the Gini index (representing inequality, including the level of income, among people in a country) ranges from 44.4 (The Phillipines, year 2015) to 32.9 (Japan, year 2013). The lower the Gini index, the more equal the people are. In regard to the density of physicians, Indonesia has the lowest value of 0.378 per 1,000 people (2017) and Japan has the highest value of 2.412 (2016). However, Indonesia has the highest number of medical schools (86 in 2018), while Singapore has three medical schools.

For data collection, a pro-forma was developed (Appendix 1). The proforma gathered information to understand the unique situation and needs of each country vis-à-vis its admission policies and methods. A leading member of the medical education community in each country was assigned to fill-in the pro-forma. This member mainly used the national level documents available in that country to fill-in the pro-forma. Since the member who filled the pro-forma was also a co-author of this paper, they were familiar with the study aims and the information expected to be documented. However, when the existing national-level documentation was insufficient to fill a particular section in the pro-forma, these members consulted other national figures in healthcare education of that country to ensure the trustworthiness and dependability of data. The collected data were compared and contrasted to: (1) identify the commonalities and differences in the admission policies and methods used among the different countries and (2) identify the commonalities and differences in the issues (or reasons) behind the adoption of particular policies and methods. The data analysis was thus focused on the two broad themes that this review was designed to study; i.e., admission policies and admission methods. Within each of these broad themes, the data collected were tabulated to identify the similarities and differences (or uniquenesses). Since the existing literature did not indicate any conceptual framework to decide which were the would-be similarities and differences within each broad theme, the constast comparative method was used for data analysis. This is because this method allowed any similarity or difference to emerge from the data. Based on the commonalities and differences that emerged and the policies and methods that different countries adopted to overcome particular issues, some common guidelines useful to future policymakers and medical schools were identified.

\section{Results}

\section{Admission policies}

\section{1) Indonesia}

Medical education in Indonesia is governed by the Ministry of Education and Culture (MoEC), in $\mathrm{Co}^{-}$ operation with the Indonesian Medical Council and the Ministry of Health. Admission to public medical schools is governed by the regulations set by the MoEC. The main admission route to enter public universities is through the joint national admission programme, which is held once a year and makes up the majority of student quotas for universities. This programme consists of two streams, the non-test path (invitation track), in which candidates are selected based on their high school academic achievement and the written test path, in which candidates must go through a series of scholastic 
aptitude and science subject tests. There are other routes, such as local university selection processes, which are at the discretion of the university. In the local admission system, each university can choose to use other selection methods, for example by adding an interview to assess candidates' non-academic attributes. There are special quotas for candidates from remote and less-developed regions. The admissions methods used by private medical schools are developed by each medical school [6]. All medical schools in Indonesia admit high school leavers.

\section{2) Japan}

The Ministry of Education, Culture, Sports, Science and Technology is the regulating body responsible for the 82 medical schools in Japan. Medical schools in Japan usually take high school leavers, though some have graduate-entry programmes [7]. Enrollment by the regional quota admission system has increased during the last 10 years for 70 out of 82 medical schools. This has been one of the largest of national policies implemented to redress the maldistribution of physicians. The number of quota entrants exceeded 1,500 per year, amounting to $17 \%$ of all medical school entrants in Japan [8]. Recently, Tokyo Medical University was found to have manipulated entrance examination scores to enroll more male students 1 . This is one reported incident of gender discrimination against female students and doctors in Japan [9].

\section{3) Malaysia}

Selection criteria for Malaysian medical schools are determined by the Malaysian Medical Council, governed by the Malaysian Medical Act 1971. The central agency for ensuring the implementation of selection criteria and standards is the Ministry of Higher Education, which provides quotas for the number of students per intake to medical schools. The academic entry requirements are

1) https://www.theguardian.com/world/2018/aug/08/tokyo-medical-s chool-admits-changing-results-to-exclude-women based on a variety of certifications, including the local Sijil Tinggi Persekolahan Malaysia, the Foundation in Science programme and the Malaysian Matriculation Programme as well as international qualifications like the General Certificate of Education (GCE) Advanced Level (A/L), International Baccalaureate, Australian Matriculation, or Unified Examination Certificate. The diversity of pre-university qualifications reflects the demographics of students entering public and private universities in Malaysia. There is an ethnicity-based selection preference in university admissions, especially in the public sector $[10,11]$. The growth in the number of medical schools in Malaysia in the past 15 years has led to an increasing number of local graduates. In tandem with returning Malaysian graduates from overseas recognised universities, there are challenges in providing house officer and medical officer jobs for junior doctors. Hence, there are calls to increase the entry requirements into medical schools for Malaysians as a measure to reduce the number of eligible candidates.

\section{4) The Philippines}

The requirements and procedures for admission to Philippine medical schools are based on the "Updated policies, standards and guidelines for medical education" (Commission on Higher Education Memorandum Order No. 18, 2016). A baccalaureate degree and taking the National Medical Admission Test are pre-requisites for applying for medical school. The selection of applicants based on non-cognitive as well as cognitive attributes is a stated principle that the medical schools should follow.

\section{5) Singapore}

The different strategies that the three medical schools in Singapore use are driven by the policy of assessing both cognitive and non-cognitive abilities of candidates [12]. The admission to the oldest medical school in Singapore, National University of Singapore, was formerly based on pre-entry cognitive ability [13], but 
has now included other non-cognitive measures. The other medical school with undergraduate entry also accepts various pre-university qualifications for admission (described under 'admission methods'). For the graduate-entry medical school, the applicants are expected to have completed (or be in the final year of) a bachelor or honours degree. Although the selection criteria differ from school to school, the Ministry of Health and the Singapore Medical Council require medical schools to formulate clear, objective, nondiscriminative selection policies aligned with the school's vision and mission and expected qualities of graduates as stipulated in the 'Outcomes and Standards for Undergraduate Medical Education in Singapore' [14] and Quality Improvement and Quality Assurance Framework.

\section{6) Sri Lanka}

The policy for admission to medical schools in Sri Lanka is based on the principles of merit and equity. Securing admission to a government medical school is highly competitive. The district quota system in Sri Lanka is an attempt to ensure equity of access to higher education. This system, which follows the principle of affirmative action, is based on the assumption that different opportunity levels for education exist across the economically diverse administrative districts of the country. The overall admission policy, which is purely based on students' performance during GCE A/L, allocates $40 \%$ of available places to 'all island merit'. This is based on the rank order of $\mathrm{z}$ scores of all the students who sat the $\mathrm{A} / \mathrm{L}$ examination during a given year for a given subject stream, in this case, biology. Of the remaining $60 \%$ of available places, $55 \%$ are distributed among all the administrative districts of the country in the form of quotas according to the estimated midyear population of the district. The remaining $5 \%$ of the opportunities are distributed across 16 pre-identified ‘educationally disadvantaged’ districts [15].

\section{7) Taiwan}

Between 1954 and 1994, students enrolling in colleges in Taiwan were required to sit the Joint College Entrance Examination. In 1994, the College Entrance Examination Center implemented the General Scholastic Ability Test (GSAT), ushering in an era of multiple pathways to college admission. The college admission process has undergone minor modifications since 1998. Currently, students planning to continue their education have three options. These are described further under admission methods. Although there are multiple options available to prospective medical students and medical school admission in Taiwan is highly competitive, to the credit of the authorities (i.e., TMAC - the Taiwan Medical Accreditation Council) the admission policies remain tightly controlled to maintain the quality of medical education. Only students within the 93rd percentile of GSAT scores gain eligibility for an interview for medical school selection (the 93rd percentile cut off was based on retrospective statistical findings). All 12 medical schools offer secondary school leaver entry programmes, and one school also offers a graduate-entry programme.

As shown by the above descriptions, the admission policies of these countries share some commonalities and many differences. One prominent commonality is the existence of a national body that determines admission policies. Usually, the national bodies implement these policies through national or common selection $\mathrm{ex}^{-}$ aminations and national guidelines issued to medical schools. However, in certain countries (e.g., Indonesia), medical schools, especially non-governmental schools, are allowed to develop their own selection system. Another commonality is the emphasis placed on merit by all countries. Some countries (e.g., Singapore) select solely based on merit, while others (e.g., Sri Lanka, Indonesia) base their selections on both merit and affirmative action. Where preferential privileges are 
Fig. 1. The Continuum of Medical Student Admission Policies

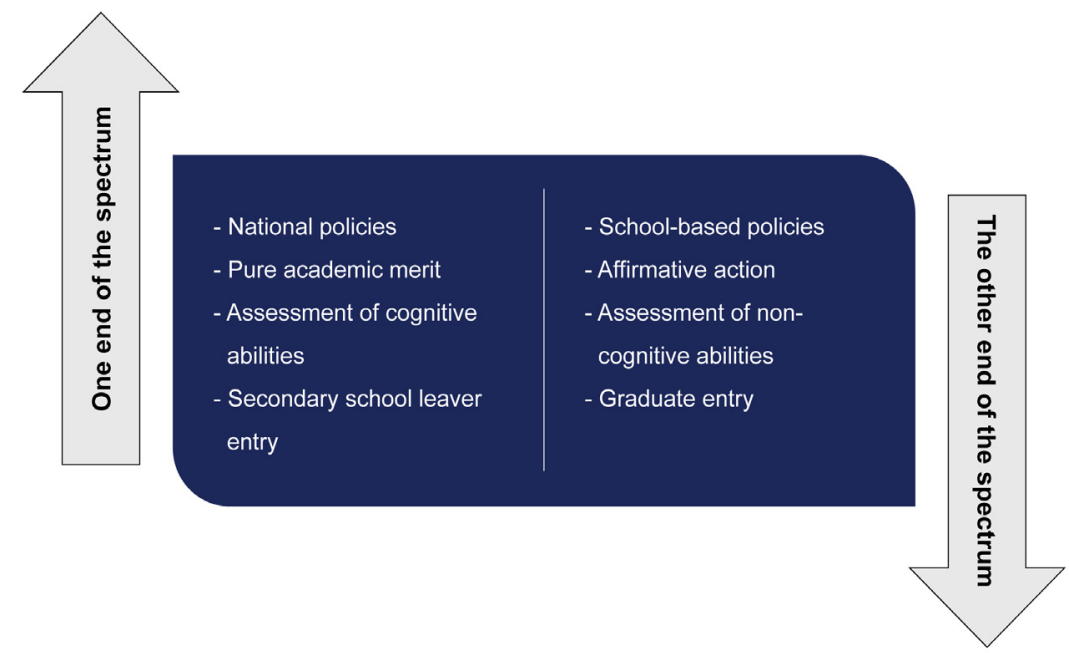

The arrow can go the other way around for both ends of the spectrum - the above direction is meant only for illustration.

afforded to certain segments of the society, the quota system is the predominantly used strategy (e.g., Japan, Malaysia, Sri Lanka). Similarly, some countries assess both cognitive and non-cognitive abilities at selection (e.g., Singapore, Taiwan) while others (e.g., Sri Lanka) assess only cognitive abilities. Certain countries have medical schools that admit both secondary school leavers and graduates (e.g., Singapore, Japan), while some others (e.g., Indonesia, Sri Lanka) admit only secondary school leavers. Each option has its own pros and cons; for example, graduate students are perhaps more motivated and mature but they may face extra burdens in terms of financial and family commitments [16,17]. Hence, policymakers need to grapple with diametrically opposite options when determining admission policies (Fig. 1).

The above policy options can be viewed on a spectrum where the two extreme considerations are indicated at either end of each option. The responsibility of the policymakers is to find the point along each spectrum that best suits the needs of the country. In doing so, they have to consider the specific socio-demographic, cultural, and economic healthcare, educational aspirations, and requirements of the country, along with the best- evidence practices available in the literature.

\section{Admission methods}

Table 1 provides an overview of the different methods that different countries use for selection of students to study medicine. Comments have also been included in a separate column to explain the use or non-use of certain methods and the issues involved.

Medical schools in most countries consider secondary school leaving examination results or final undergraduate examination results (e.g., cumulative grade point average) to determine the academic ability of the candidate. Some countries (e.g., Indonesia, Japan, the Philippines) have national entry examinations to assess academic ability. These examinations are usually aptitude tests, which are standardised tests to measure a person's aptitude for learning [18]. In addition, some countries, like Indonesia, Malaysia, and Singapore, use both high school examination results and aptitude test results for selection.

With regard to non-academic or non-cognitive abilities, a few schools use newer methods like Situational Judgment Tests (SJTs) and MMIs. That said, some countries use traditional interviews or do not use any 


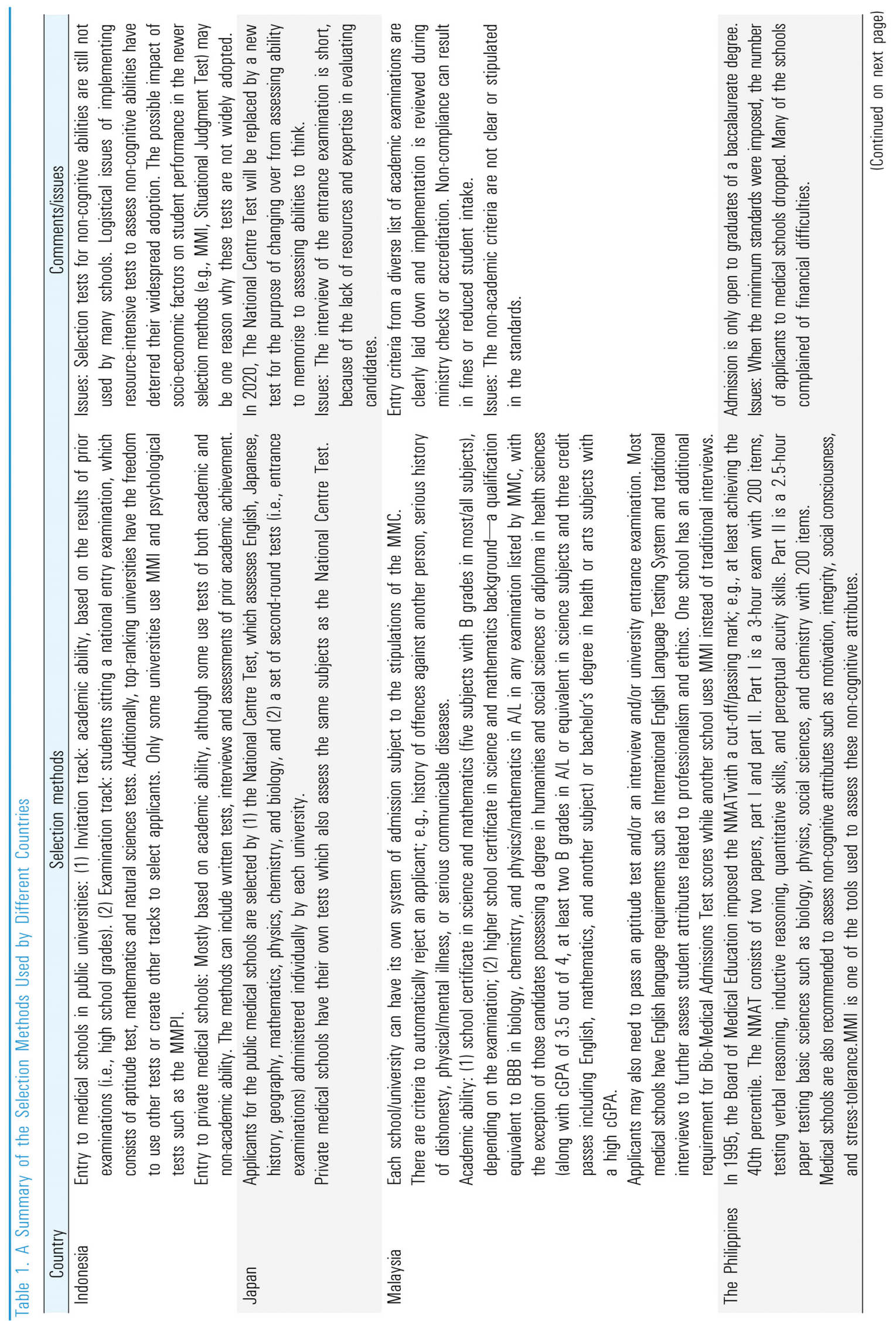




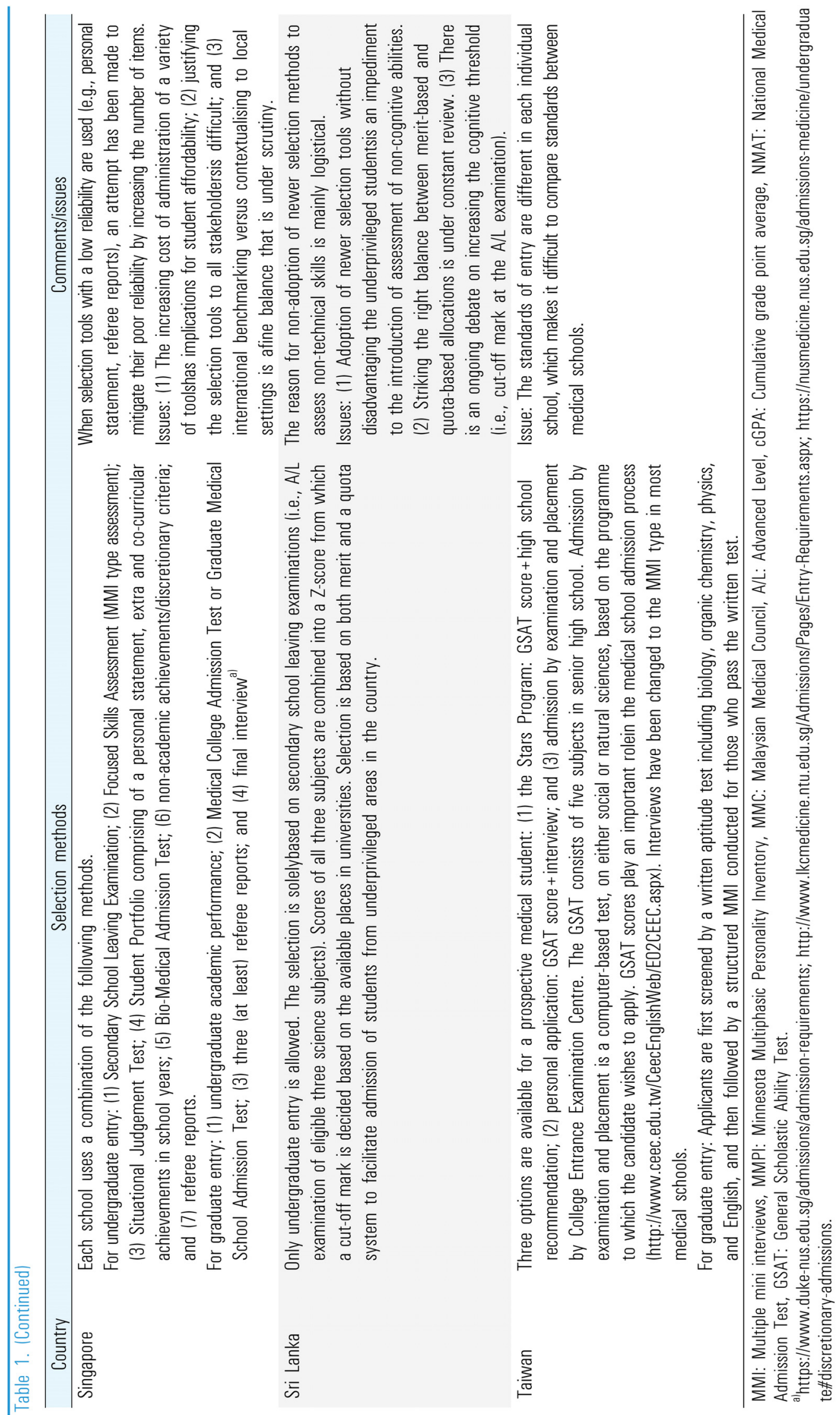


method to assess non-academic abilities. The main reason for the non-use of newer methods of admission in all countries is resource limitations. Another, more country-specific reason for the non-adoption of newer admission methods is the concern that these methods may adversely affect the opportunities for marginalised sections of the society.

\section{Discussion}

Finding the right policies and methods for admission to medical education is a balancing act, often dependent on the healthcare system needs of the country. While the admission process needs to be fair to society by selecting the applicants with the potential to be good doctors, it also needs to be fair to the applicants, who are often a diverse group of individuals unified by their common desire to become doctors. Admission policies and methods, hence, must also include special schemes to promote the admission of applicants from underrepresented groups, such as minority groups, students from rural areas, and underprivileged families whose performance in selection examinations may be affected for various socio-economic reasons [19,20]. Taiwan's Stars Program, for example, has been developed to promote the admission of students from rural areas and underprivileged families. Despite the limited number of countries that were reviewed, we were able to capture a broad range of admission policies and methods with different characteristics that suit varying socioeconomic conditions, population/country needs, and educational best practices. However, this study identified tension between many of these policies and methods. Below is an exploration of some of the more prominent examples of these policy-method tensions.

\section{Admission methods versus resources}

The admission methods used by the seven countries represent the most commonly reported methods in the literature, such as previous academic achievement (secondary school results), interviews, references, personal statements, and aptitude tests [21].

Medical schools have invested in selection systems to select the most appropriate candidates, those who can complete medical studies on time and are fit to practise medicine [22]. Therefore, it is reasonable to expect a high 'return on investment' (i.e., a high number of students entering medical schools complete their studies on time and practise as competent medical doctors) when using resource-intensive admission methods. In developing countries where resources are scarce, the careful use of financial and other resources is essential to ensure that these purposes are met through the best admission methods available and applicable. For example, in Indonesia, one of the barriers to implementing a resource-intensive selection test would be logistical issues, given the geography and demography of the country. This is also felt in one of the developed countries, Japan, where selection interviews face challenges due to insufficient resources. The application of methods such as MMI and SJT requires strong financial and logistical support and also an adequate number of trained human resources. This is one of the challenges faced by many countries, particularly developing countries, despite the potential advantages of those methods [23]. The availability of resources may conflict with the policy of using the most appropriate and best-evidence admission methods.

\section{Assessment of non-cognitive attributes versus widening participation}

The importance of selecting applicants based on their 
non-cognitive potential has been recognised by many medical schools, for example, in Singapore, Indonesia, the Philippines, and Taiwan. Some countries have taken a step further by including admission methods that assess non-cognitive attributes as a part of the selection system, for example, by implementing MMI and SJT. Some of these methods of admission, however, may not be politically acceptable in certain countries because they limit the participation of underrepresented minorities or applicants from underprivileged areas in the selection process. For example, in Sri Lanka, the introduction of aptitude testing, MMI and the English proficiency requirement would disadvantage many groups of applicants. Similar to Sri Lanka, Indonesia is now facing challenges in applying non-cognitive attribute-based admission methods without favouring applicants from developed provinces, who are more likely to have received coaching on these methods prior to admission. This situation is further complicated by wide gaps in the quality of secondary education in different parts of the country.

\section{Maintaining assessment standards and sustaining the medical education pro- gramme versus widening participation}

Griffin and Hu [24], in their study in Australia, found that the number of students from disadvantaged socioeconomic and socio-educational backgrounds applying to medical schools was significantly lower compared to those coming from more advantaged backgrounds. They also found that students from disadvantaged backgrounds were adversely affected by the cognitive ability test in the selection process. Mathers and Parry [25] conducted a study to understand the reasons behind the low number of working-class applicants to medical schools in the United Kingdom and found that the interplay between socio-cultural context, family and institutional per- ceptions plays a role in causing students from disadvantaged backgrounds to be less likely to pursue medicine as a career. There is still a perception in society that medicine is an 'elite' profession. Different policies have been adopted to encourage more students from underprivileged backgrounds to enter medical school. One such policy is to lower the admission bar for applicants from certain socio-economic backgrounds. This is also known as affirmative action or positive discrimination. In our sample, several countries, such as Sri Lanka, Malaysia, and Japan, have resorted to quota systems to achieve this.

However, such a policy should also ensure that the minimum standards are maintained to avoid an excessive dropout rate. This has been described as the "revolving door syndrome' [20], where both the intake and the dropout are simultaneously increased. Conversely, keeping admission standards high does not ensure that an adequate number of candidates is recruited, both to sustain the medical education programme and to fulfil the healthcare requirements of the country. Hence, it is a delicate balance to identify the minimum adequate standards for following a course in medicine without being an excessive barrier to socio-economically disadvantaged applicants, while at the same time keeping the medical school account balances afloat and satisfying the country's need for medical professionals. For example, the Philippines experienced a drop in the number of candidates when standards for selection were increased and this caused financial problems for schools that relied on students' tuition fees.

The World Health Organization endorses a capable, inclusive and tolerant medical school population as a part of medical schools' social accountability $[3,26]$. Girotti et al. [27] argued that medical schools, whether public or private, have the responsibility to be socially accountable. Therefore, there should be a balance 
between the social and 'financial' agenda of medical schools. This is particularly the case for private medical schools that are financially self-supporting.

\section{Conclusions}

Based on the above discussion, it is clear that the methods that countries adopt, by and large, depend on their national policy. However, this study found that sometimes the methods adopted to implement one policy will be detrimental to the implementation of another policy. The following are the most obvious of such policy-method conflicts.

- The adoption of newer methods to promote the policy of selecting the best possible (i.e., wellrounded) applicants for medicine (by assessing an applicant's all-round ability, including non-technical abilities) is at odds with the policy of implementing a resource-friendly admission process that all schools within a country can afford.

- The methods used to assess aptitude in noncognitive abilities at admission may interfere with the policy of widening access, as many applicants from disadvantaged backgrounds may not have access to training facilities that train applicants for non-cognitive aptitude tests.

- The methods used to select the most appropriate applicants for medicine may often clash with the policy of widening access, as lowering of the standards of selection criteria would often be necessary to accommodate applicants from disadvantaged backgrounds.

- The methods adopted to implement the policy of selecting the most appropriate applicants for medicine may conflict with the policy of producing a sufficient number of medical professionals to meet the health needs of the country (i.e., the quality versus quantity tension).

How best to customise the best practices to meet local needs is the challenge faced by all countries. Overcoming this challenge largely depends on how policymakers and administrators reconcile the inherent tension between some of the policies and methods, so that the most appropriate trade-off point in the policy continuum that this paper conceptualised can be selected. Continuous fine-tuning and re-evaluation of the policies and methods that are currently used to find the right combination of methods and policies, perhaps with the use of action research, would enable the adoption of the most productive and efficient policies and methods.

We also note that the number of studies investigating how well the adopted methods achieve the policy requirements is limited. Hence, there is a pressing need to conduct more research, not only to identify how well the existing methods achieve the policy requirements, but also to identify how best the above policy-method conflicts can be balanced. Finding the right balance requires careful consideration of all variables, including the country's socio-economic status, the school's vision and mission, the expected competencies of graduates, and the availability of resources.

\section{ORCID:}

Diantha Soemantri: https://orcid.org/0000-0002-3956-1265;

Indika Karunathilake: https://orcid.org/0000-0001-9772-116X;

Jen-Hung Yang: https://orcid.org/0000-0002-0800-8395;

Shan-Chwen Chang: https://orcid.org/0000-0001-6505-4139;

Chyi-Her Lin: https://orcid.org/0000-0001-7163-528X;

Vishna D. Nadarajah: https://orcid.org/0000-0002-7126-7189;

Hiroshi Nishigori: https://orcid.org/0000-0002-0715-7073;

Dujeepa D. Samarasekera: https://orcid.org/0000-0002-6916-6741;

Shuh Shing Lee: https://orcid.org/0000-0002-6760-1340; 
Lilybeth R. Tanchoco: https://orcid.org/0000-0002-2007-0749;

Gominda Ponnamperuma: https://orcid.org/0000-0003-0023-6550

Acknowledgements: This study was presented at the 12th Jakarta Meeting on Medical Education organized by Faculty of Medicine Universitas Indonesia, 19-20 October 2019. The authors wish to thank the Asia Pacific Medical Education Network (APME-Net), an association dedicated to promoting scholarship in medical education, under the auspices of which this study was conducted.

Funding: No financial support was received for this study. Conflicts of interest: No potential conflict of interest relevant to this article was reported.

Author contributions: DS, IK, and GP initiated and designed the study. Data was provided by all authors. All authors drafted particular parts of the manuscript, reviewed, and approved the final version of the manuscript.

\section{References}

1. Laurence CO, Zajac IT, Lorimer M, Turnbull DA, Sumner KE. The impact of preparatory activities on medical school selection outcomes: a cross-sectional survey of applicants to the University of Adelaide Medical School in 2007. BMC Med Educ. 2013;13:159.

2. Patterson F, Roberts C, Hanson MD, et al. 2018 Ottawa consensus statement: selection and recruitment to the healthcare professions. Med Teach. 2018;40(11):10911101.

3. Roberts C, Prideaux D. Selection for medical schools: re-imaging as an international discourse. Med Educ. 2010;44(11):1054-1056.

4. Martin AJ, Beska BJ, Wood G, et al. Widening interest, widening participation: factors influencing school students' aspirations to study medicine. BMC Med Educ. 2018;18(1):117.

5. O’Neill L, Vonsild MC, Wallstedt B, Dornan T.
Admission criteria and diversity in medical school. Med Educ. 2013;47(6):557-561.

6. Hidayah RN. Impact of the national medical licensing examination in Indonesia: perspectives from students, teachers, and medical schools [dissertation]. Leeds, UK University of Leeds; 2018.

7. Kozu T. Medical education in Japan. Acad Med. 2006; 8I(12):1069-1075.

8. Matsumoto M, Takeuchi K, Tanaka J, et al. Follow-up study of the regional quota system of Japanese medical schools and prefecture scholarship programmes: a study protocol. BMJ Open. 2016;6(4):e011165.

9. Matsui T, Sato M, Kato Y, Nishigori H. Professional identity formation of female doctors in Japan: gap between the married and unmarried. BMC Med Educ. 2019;19(1):55.

10. Yusoff MS, Rahim AF, Baba AA, Esa AR. Medical student selection process and its pre-admission scores association with the new students' academic performance in Universiti Sains Malaysia. Int Med J. 201 1;18(4): 327-331.

11. Arzuman H, Ja'afar R, Fakri NM. The influence of pre-admission tracks on students' academic performance in a medical programme: Universiti Sains Malaysia. Educ Health (Abingdon). 2012;25(2):124-127.

12. Samarasekera DD, Ooi S, Yeo SP, Hooi SC. Medical education in Singapore. Med Teach. 2015;37(8):707713.

13. Wong JE. The future of medical education: the second 100 years. Ann Acad Med Singapore. 2005;34(6):166C$171 \mathrm{C}$.

14. Ministry of Health. Outcomes and standards for undergraduate medical education in Singapore: recommendations of the National Medical Undergraduate Curriculum Committee. Singapore: Ministry of Health; 2014.

15. Mendis L, Karunathilake I, Ponnamperuma G. Admission of Sri Lankan students for medical education: time 
to review. Ceylon Med J. 2018;63(2):49-52.

16. Peile E. Should all medical students be graduates first? Yes. BMJ. 2007;335(7629):1072.

17. George C. Should all medical students be graduates first? No. BMJ. 2007;335(7629):1073.

18. Patterson F, Ferguson E. Selection for medical education and training. In: Swanwick T, ed. Understanding Medical Education: Evidence, Theory and Practice. Oxford, UK: Wiley Blackwell; 2010:359.

19. Hughes P. Can we improve on how we select medical students? J R Soc Med. 2002;95(1):18-22.

20. Tutton P, Price M. Selection of medical students. BMJ. 2002;324(7347):1170-1171.

21. Ferguson E, James D, Madeley L. Factors associated with success in medical school: systematic review of the literature. BMJ. 2002;324(7343):952-957.

22. Edwards D, Friedman T, Pearce J. Same admissions tools, different outcomes: a critical perspective on predictive validity in three undergraduate medical schools. BMC Med Educ. 2013;13:173.

23. Bassaw B, Pitt-Miller P. Modernizing medical education: perspective from a developing country. West Indian Med J. 2007;56(1):80-85.

24. Griffin $\mathrm{B}, \mathrm{Hu} \mathrm{W}$. The interaction of socio-economic status and gender in widening participation in medicine. Med Educ. 2015;49(1):103-113.

25. Mathers J, Parry J. Why are there so few working-class applicants to medical schools?: learning from the success stories. Med Educ. 2009;43(3):219-228.

26. Boelen C, Heck J. Defining and measuring the social accountability of medical schools. Geneva, Switzerland: World Health Organization; 1995.

27. Girotti JA, Park YS, Tekian A. Ensuring a fair and equitable selection of students to serve society's health care needs. Med Educ. 2015;49(1):84-92. 
Diantha Soemantri, et al: Medical school admission in seven Asian countries

Appendix 1. The Pro-forma for Data Collection to Be Completed by the Contributor from Each Country

Name of the country:

\begin{tabular}{l}
\hline \multicolumn{1}{c}{ Component } \\
\hline Overview-context \\
Current medical education situation \\
National policy \\
Methods used \\
Issues/challenges \\
Any unique aspects
\end{tabular}

